\title{
Rapid melt crystallization of amorphous-silicon thin films
}

\author{
Fude Liu, ${ }^{\text {a) }}$ Lei Wang, Guandong Yang, and Wentao Wang \\ Department of Mechanical Engineering, The University of Hong Kong, Pokfulam Road, Hong Kong
}

(Received 26 December 2012; accepted 18 February 2013; published online 1 March 2013)

\begin{abstract}
We investigated the potential to melt crystallize amorphous $\mathrm{Si}$ thin films on glass with electromagnetic irradiation at $13.56 \mathrm{MHz}, 2.45 \mathrm{GHz}$, and $110 \mathrm{GHz}$, respectively. We showed that the melt crystallization of millimeter-wave annealing could be an ideal choice for achieving highquality semiconductor thin films with thickness in the micron range. In particular, silicon pillars with large ultra-high crystalline quality grains were experimentally realized by millimeter-wave annealing amorphous silicon on glass. Our simulation results were consistent with the experimental ones. Such understandings may enable us to prepare high-quality thin films on inexpensive substrates for solar cells and other solid-state devices. (c) 2013 American Institute of Physics. [http://dx.doi.org/10.1063/1.4794085]
\end{abstract}

High-quality polycrystalline silicon (poly-Si) thin films on inexpensive foreign substrates have many potential applications, especially for high-performance solar cells and highmobility thin-film Si transistors. ${ }^{1}$ However, it has been shown to be extremely challenging to grow high-quality poly-Si on those substrates such as glass, plastic, and stainless steel. ${ }^{2}$ One major issue is that inexpensive substrates generally cannot be processed under high temperatures $\left(>600^{\circ} \mathrm{C}\right)$. Low-temperature, long-time solid-phase crystallization (SPC) of amorphous $\mathrm{Si}(a-\mathrm{Si})$ is often used to prepare poly-Si films that in general contain either intolerantly high levels of intragrain defects or impurities. ${ }^{3}$ It is well known that silicon crystallized from the liquid melt has high crystalline quality, for example, in the case of silicon wafers prepared with the Czochralski (CZ) growth process. ${ }^{4}$ Although laser annealing has been extensively studied in melt crystallizing $a$-Si, there are still some issues that include the difficulty in precisely controlling material characteristics with this technology. ${ }^{5}$ So it is worth exploring new annealing approaches. Ideally, the energy absorption during annealing should be limited to the thin film with minimum absorption in the substrate, and the energy transfer rates from the radiation source to the Si film should be very high. In this study, we showed that millimeter-wave pulse annealing on millisecond time scales could be an ideal choice to meet these criteria, with advantages over traditional centimeter-wave for material processing. ${ }^{6,7}$

A $2-\mu$ m thick $a$-Si thin film was deposited onto a glass substrate (Fig. 1(a)). The substrate was coated with a $\mathrm{SiO}_{2}$ barrier layer (radiation transparent) of $\sim 100 \mathrm{~nm}$ before the deposition of $a$-Si. $\mathrm{SiO}_{2}$ was also used as the capping layer ( $\sim 200 \mathrm{~nm}$ ). Samples were first annealed at $540^{\circ} \mathrm{C}$ to remove $\mathrm{H}$ from the $a$-Si, while not crystallizing the $a$-Si. The sample was then put in a vacuum chamber to be melt crystallized with electromagnetic radiation. The experimental setup of the radiation annealing is shown schematically in Fig. 1(b). The sample was illuminated with a radiation source at a certain power density. An optical pyrometer was used to monitor the

\footnotetext{
a) Author to whom correspondence should be addressed. Electronic mail: fordliu@hku.hk.
}

sample temperature by detecting the radiation in the optical or near-infrared spectrum from the sample. We experimentally studied samples illuminated on an area of $\sim 1 \mathrm{~cm}^{2}$ with $110-\mathrm{GHz}$ radiation single pulse from a 1-MW gyrotron. ${ }^{8,9}$ The power density at the sample was $\sim 40 \mathrm{~kW} / \mathrm{cm}^{2}$. Typical pulse lengths were $1-8.5 \mathrm{~ms}$ in duration. More experimental details can be found elsewhere. ${ }^{8,10}$ Calculations on the interaction between the radiation and thin films at three typical frequencies $(13.56 \mathrm{MHz}, 2.45 \mathrm{GHz}$, and $110 \mathrm{GHz}$ ) were carried out, respectively, with the computational software program MATHEMATICA ${ }^{\circledR}$.

The experimental results of $a$-Si samples annealed with $110 \mathrm{GHz}$ millimeter wave are shown in Fig. 2. Fig. 2(a) shows the scanning electron microscopy (SEM) image of a typical post-annealed $\mathrm{Si}$ film taken at a tilt angle of $52^{\circ}$ for a better 3D view. Most pillars appear to be bare silicon (uncovered), whereas others are coated with the capping $\mathrm{SiO}_{2}$ film (covered). High-magnification SEM images taken from uncovered and covered pillar regions are depicted in Figs. 2(b) and 2(c), respectively. During the short singlepulse anneals, the $a$-Si film melted (its melting point is in the (a)

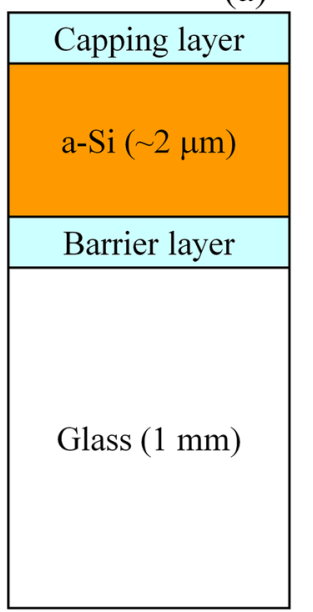

(b)

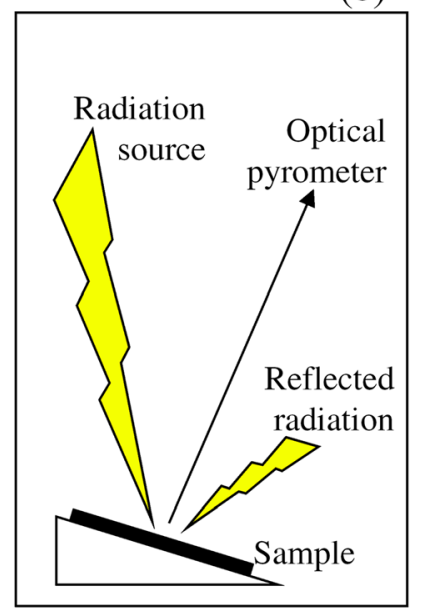

FIG. 1. Electromagnetic radiation annealing of $a-\mathrm{Si}$ on $\mathrm{SiO}_{2} /$ glass . (a) Schematic of the sample structure before annealing. (b) Schematic of the experimental setup of the radiation annealing. 

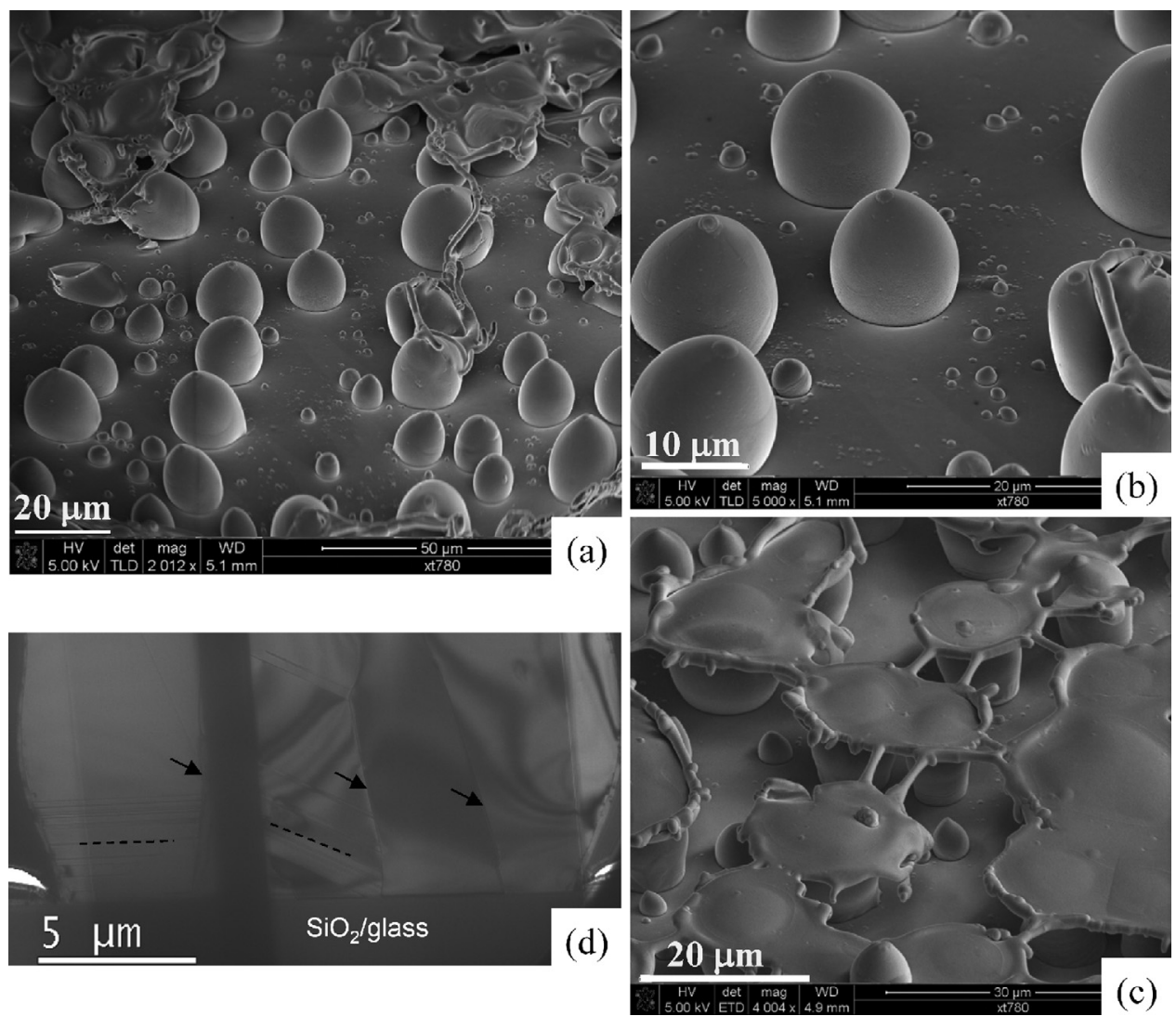

FIG. 2. Microscopy images of ultrahighcrystalline-quality $\mathrm{Si}$ pillars formed by millimeter-wave annealing of $a$-Si on glass. (a) Low-magnification SEM image of the sample after millimeter-wave annealing (all SEM images shown here were taken at a sample tilt angle of $52^{\circ}$ for a better 3D view). (b) High-magnification SEM image taken from an uncovered pillar region. (c) High-magnification SEM image taken from a covered pillar region. (d) Cross-sectional TEM image of a Si pillar. The arrows and dashed lines indicate the grain boundaries and $\Sigma 3\langle 111\rangle$ twins, respectively. range of $\sim 1000-1450{ }^{\circ} \mathrm{C}$ ) and formed crystalline silicon ( $c$-Si). The melted film balled up on the glass substrate, and silicon pillars crystallized from the liquid melt. A typical cross-sectional transmission electron microscopy (TEM) image of a specimen sectioned from a pillar is shown in Fig. 2(d). These pillars are usually composed of 1-4 large grains (with grain size up to $20 \mu \mathrm{m}$ ). In addition, the grains have ultra-high crystalline quality with only $\Sigma 3\langle 111\rangle$ twins observed mainly close to the substrate, and the grain boundaries (and growth direction) are perpendicular to the substrate surface, as indicated by the dashed lines and arrows, respectively. In the following, we investigate the interaction of radiation with intrinsic $\mathrm{Si}$ at $13.56 \mathrm{MHz}, 2.45 \mathrm{GHz}$, and $110 \mathrm{GHz}$, respectively.

The electrical conductivity $\sigma$ plays an important role in the energy absorption of the Si film during radiation annealing. The electrical conductivity $\sigma$ of a semiconductor can be expressed as follows: ${ }^{11}$

$$
\sigma=c T^{1.5} q\left(\mu_{e}+\mu_{h}\right) \exp \left(-\frac{E_{g}}{2 k_{B} T}\right),
$$

where $c$ is a constant, $T$ is the temperature, $q$ is the elementary charge, and $k_{B}$ is the Boltzmann constant. The temperaturedependence of electron and hole mobilities $\left(\mu_{e}, \mu_{h}\right)$ and energy bandgap $\left(E_{g}\right)$ can be modeled with the empirical relationships ${ }^{12}$

$$
\begin{gathered}
\mu_{e}=92\left(\frac{T}{300}\right)^{-0.57}+1268\left(\frac{T}{300}\right)^{-2.33}, \\
\mu_{h}=54.3\left(\frac{T}{300}\right)^{-0.57}+406.9\left(\frac{T}{300}\right)^{-2.23},
\end{gathered}
$$

$$
E_{g}=1.17-\frac{4.73 \times 10^{-4} T^{2}}{T+636}
$$

We estimated approximately the constant $c=6.87 \times 10^{15}$ $\mathrm{cm}^{-3} \mathrm{~K}^{-3 / 2}$ by least-squares fitting the conductivity $\sigma$ expression with experimental data for the variation of conductivity with temperature for intrinsic $c$-Si given in the literature. ${ }^{13} \mathrm{We}$ then plotted directly the electrical conductivity $\sigma$ as a function of temperature $T$. The log-plot is shown in Fig. 3(a).

In order to have the optimal power transfer from free space to the conducting medium (in this case, the silicon film), it requires that the impedances closely match with each other. The magnitude of the impedance for a good conducting medium is expressed by the following equation: ${ }^{14}$

$$
|Z|=\sqrt{\frac{\mu_{0} \omega}{\sigma}}
$$

where $\mu_{0}$ is the magnetic permeability of free space $\left(=4 \pi \times 10^{-7} \mathrm{H} / \mathrm{m}\right)$ and $\omega$ is the angular frequency. The value of wave impedance in free space $\mathrm{Z}_{0}$ is $\sim 377 \Omega$. According to the literature, ${ }^{15}$ the static wave impedance of $\mathrm{SiO}_{2}$ was estimated to be $190.85 \Omega$. It is obvious that adding a $\mathrm{SiO}_{2}$ capping layer enhances coupling the electromagnetic fields into the $\mathrm{Si}$. To simplify things, however, we ignore the $\mathrm{SiO}_{2}$ capping layer in the following discussion, i.e., we consider only direct interaction between the radiation and the $\mathrm{Si}$ layer. Since we already know the relationship between the conductivity $\sigma$ and temperature $\mathrm{T}$, the plot of $Z / Z_{0}(=\alpha)$ as a function of temperature at different frequencies can be readily obtained, as shown in Fig. 3(b). At high temperatures $(>600 \mathrm{~K})$, we see that the values of $\alpha$ are much smaller at 

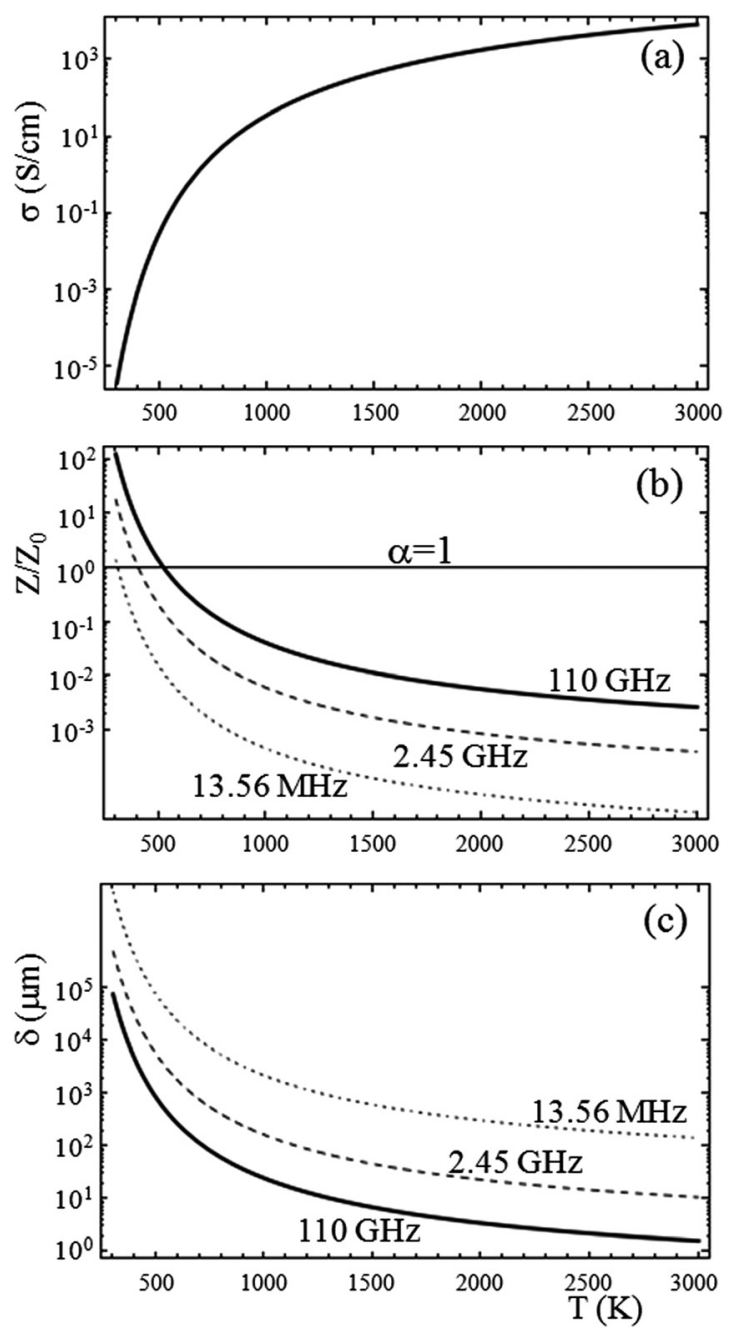

FIG. 3. Different parameters vs. temperature. (a) Electrical conductivity as a function of temperature. (b) Impedance as a function of temperature at $13.56 \mathrm{MHz}, 2.45 \mathrm{GHz}$, and $110 \mathrm{GHz}$, respectively (the $\alpha=1$ line is included for easy comparison). (c) Skin depth as a function of temperature at 13.56 MHz, $2.45 \mathrm{GHz}$, and $110 \mathrm{GHz}$, respectively.

2.45 GHz and $13.56 \mathrm{MHz}$ than at $110 \mathrm{GHz}$, although the impedance match is better at lower frequencies and temperatures. In fact, in the limit of $\alpha \ll 1$ (i.e., Si becomes a socalled good conductor now), the coefficient $\mathrm{R}$ of reflection becomes, $R \approx 1-2 \sqrt{2} \alpha$, by considering the continuity across the interface. ${ }^{16}$ So most of the incident energy is reflected and only a small fraction of energy is absorbed by the Si film at low frequencies of $13.56 \mathrm{MHz}$ and $2.45 \mathrm{GHz}$ when the temperature is greater than $600 \mathrm{~K}$. In comparison, the impedances better match for each other at $110 \mathrm{GHz}$ in the high temperature range $(>600 \mathrm{~K})$. Therefore, we see that the power transfer is much higher at $110 \mathrm{GHz}$ than at $2.45 \mathrm{GHz}$ and $13.56 \mathrm{MHz}$, which can be further confirmed later in Fig. 4.

Another important parameter we need to consider is the skin depth $\delta(\delta=\sqrt{2 /(\omega \mu \sigma)}$, where $\mu$ the absolute magnetic permeability $\left(=0.9999 \mu_{0}\right.$ for $\left.\left.\mathrm{Si}\right)\right){ }^{14}$ Similarly, because we already knew the relationship between the conductivity $\sigma$ and temperature $\mathrm{T}$, the skin depth $\delta$ as a function of temperature at different frequencies can be readily plotted (Fig. 3(c)), assuming other material properties are not themselves frequency-dependent. At $2.45 \mathrm{GHz}$ and $13.56 \mathrm{MHz}$, the
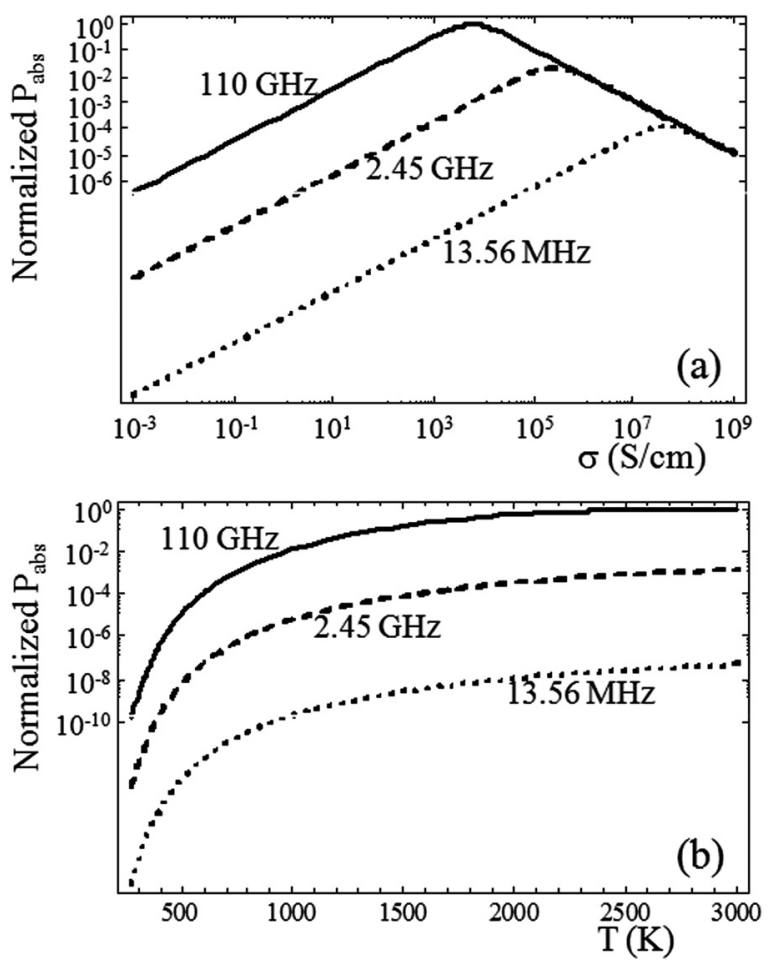

FIG. 4. Power absorption vs. conductivity (or temperature). (a) Normalized power absorption as a function of conductivity. (b) Normalized power absorption as a function of temperature. In comparison, included are those plots at $2.45 \mathrm{GHz}, 13.56 \mathrm{MHz}$, and $110 \mathrm{GHz}$, respectively.

skin depth is much greater than the Si film thickness $(\sim 2 \mu \mathrm{m})$ in all temperature ranges shown here $(300-3000 \mathrm{~K})$. On the other hand, the skin depth would be less than $1 \mu \mathrm{m}$ if the laser or lamp-based anneals were applied, in particular at temperatures above $600 \mathrm{~K}$. Ideally, the skin depth is expected to be close to the Si film thickness, in particular when it is in the high temperature range, which ensures that radiation absorption is mainly limited to $\mathrm{Si}$. We can see that $110 \mathrm{GHz}$ millimeter-wave is an ideal choice to anneal the whole Si film of a few microns thick especially in the high temperature range as far as the skin depth is concerned.

With the above knowledge at hand, we can now analyze the total power absorption in the film with the expression, ${ }^{17}$

$$
P_{a b s}=\frac{\pi a^{2} t_{S i}{ }^{3}}{\delta^{4} \sigma} H_{0}^{2} /\left(1+\left(\frac{t_{S i}}{\delta}\right)^{4}\right),
$$

where $H_{0}$ is the magnitude of the magnetic field, $t_{S i}$ is the $\mathrm{Si}$ film thickness (let $t_{S i}=2 \mu \mathrm{m}$ for this work), and $\pi a^{2}$ ( $a=$ radius) is the sample surface area irradiated. The normalized power absorption as a function of conductivity is then plotted in Fig. 4(a), and its corresponding plot as a function of temperature is shown in Fig. 4(b). In comparison, included are those plots at $110 \mathrm{GHz}, 2.45 \mathrm{GHz}$, and $13.56 \mathrm{MHz}$, respectively. For all cases, we see that the power absorption increases with temperature until reaching a peak value and then decreases slightly. The peak position moves to a higher conductivity/temperature value as the frequency decreases. The power absorption is eventually the same for all cases at a much higher temperature that is well above the boiling point of $\mathrm{SiO}_{2}(2500 \mathrm{~K})$. However, we are more concerned with the temperature in the range of $300-2500 \mathrm{~K}$, i.e., the 
room temperature to the boiling point of $\mathrm{SiO}_{2}$. The power absorption at $110 \mathrm{GHz}$ is a few orders higher than that at $2.45 \mathrm{GHz}$ and $13.56 \mathrm{MHz}$ in this temperature range, which explains why we can directly apply $110 \mathrm{GHz}$ millimeterwave to melt crystallize intrinsic $a$-Si without using a susceptor or a resonant cavity to assist the radiation absorption as in the cases of $2.45 \mathrm{GHz}$ and $13.56 \mathrm{MHz}{ }^{17,18}$

Because the optical constants of $a$-Si for $110 \mathrm{GHz}$ radiation are not known, the exact percentage of the incident power density absorbed by the Si film still is not well characterized. However, we can estimate approximately the total energy absorbed. As seen from Fig. 2, the capping $\mathrm{SiO}_{2}$ still stayed on the top after annealing although it lost its integrity, while the wetting $\mathrm{SiO}_{2}$ on glass remained its integrity. With the knowledge at hand, we can reasonably assume that the peak average temperature of the Si film is higher than the melting point of $\mathrm{SiO}_{2}(1873-1998 \mathrm{~K})$, but unlikely to be above its boiling point $(2500 \mathrm{~K})$. Considering that typical pulse lengths are $1-8.5 \mathrm{~ms}$ in duration, we can derive that the heating rates are on the order of $10^{6} \mathrm{~K} / \mathrm{s}$, which corroborates well the simulated results by Thompson et al. ${ }^{7}$ The net power absorbed by the Si film can be estimated approximately by using the equation

$$
P_{a b s}=M C_{p} \frac{\partial T}{\partial t}
$$

where $M$ is the material mass (mass density $\rho=2.33 \mathrm{~g} / \mathrm{cm}^{3}$ for $\mathrm{Si}), C_{p}$ is the specific heat $(0.71 \mathrm{~J} / \mathrm{g} \cdot \mathrm{K}$ for $\mathrm{Si})$, and $t$ is the time. We can get $P_{a b s} \approx 330 \mathrm{~W} / \mathrm{cm}^{2}$ at a heating rate of $10^{6} \mathrm{~K} / \mathrm{s}$ for a $2 \mu \mathrm{m}$-thick $\mathrm{Si}$ film. The value can at least be doubled, i.e., $P_{a b s} \approx 660 \mathrm{~W} / \mathrm{cm}^{2}$, after accounting for heat losses from the Si film via conduction and radiation. The net power-absorption magnitude is significant considering the thickness of a few $\mu \mathrm{m}$ only and the very short pulse lengths.

In summary, we investigated the potential to melt crystallize amorphous Si thin films on glass with electromagnetic irradiation at three different frequencies $(13.56 \mathrm{MHz}$, $2.45 \mathrm{GHz}$, and $110 \mathrm{GHz}$ ). The interaction of radiation with intrinsic Si was studied both experimentally and theoretically in detail. Silicon pillars with large ultra-high crystalline quality grains were experimentally realized by millimeter-wave annealing of amorphous silicon on glass. In addition, our simulation results were consistent with the experimental ones. We, therefore, showed that the melt crystallization of high frequency millimeter-wave annealing could be an ideal choice for achieving high-quality semiconductor thin films with thickness in the micron range. Such understandings may enable us to prepare high-quality semiconductor thin films on inexpensive foreign substrates for high-performance solar cells and other solid-state devices.

The authors thank the financial support from the Seed Funding Programme for Basic Research at HKU (Project Code 200910159016), the University Development Fund (UDF) 2009-2010 (Second Round), and the HKU Initiative on Clean Energy \& Environment (HKU-ICEE). Also, thanks go to the National Renewable Energy Laboratory (NREL) for access to the microscopy facilities. Last but not least, the authors thank greatly D. L. Young from NREL for providing Si samples annealed with $110 \mathrm{GHz}$ millimeter wave.

${ }^{1}$ G. Beaucarne, Adv. OptoElectron. 2007, 12; M. A. Green, Sol. Energy 74, 181 (2003); A. G. Aberle, Thin Solid Films 511-512, 26 (2006); A. Shah, P. Torres, R. Tscharner, N. Wyrsch, and H. Keppner, Science 285, 692 (1999).

${ }^{2}$ A. G. Aberle, J. Cryst. Growth 287, 386 (2006); I. Gordon, D. Van Gestel, K. Van Nieuwenhuysen, L. Carnel, G. Beaucarne, and J. Poortmans, Thin Solid Films 487, 113 (2005); C. W. Teplin, D. S. Ginley, and H. M. Branz, J. Non-Cryst. Solids 352, 984 (2006).

${ }^{3}$ F. Liu, M. J. Romero, K. M. Jones, A. G. Norman, M. M. Al-Jassim, D. Inns, and A. G. Aberle, Thin Solid Films 516, 6409 (2008); M. L. Terry, A. Straub, D. Inns, D. Song, and A. G. Aberle, Appl. Phys. Lett. 86, 172108 (2005).

${ }^{4}$ J. D. Plummer, M. D. Deal, and P. B. Griffin, Silicon VLSI Technology: Fundamentals, Practice, and Modeling (Prentice Hall, 2000), pp. 93-146.

${ }^{5} \mathrm{~J}$. Perriere, E. Millon, and E. Fogarassy, Recent Advances in Laser Processing of Materials, 1st ed. (Elsevier Science, 2006), p. 317.

${ }^{6}$ K. J. Rao, B. Vaidhyanathan, M. Ganguli, and P. A. Ramakrishnan, Chem. Mater. 11, 882 (1999); C.-J. Ko, Y.-K. Lin, and F.-C. Chen, Adv. Mater. 19, 3520 (2007); S. G. Sundaresan, M. Murthy, M. V. Rao, J. A. Schreifels, M. A. Mastro, C. R. Eddy, Jr., R. T. Holm, R. L. Henry, J. A. Freitas, Jr., E. Gomar-Nadal, R. D. Vispute, and Y.-L. Tian, Semicond. Sci. Technol. 22, 1151 (2007); Y. Makino, ISIJ Int. 47(4), 539 (2007).

${ }^{7}$ K. Thompson, J. H. Booske, R. L. Ives, J. Lohr, Y. Gorelov, and K. Kajiwara, J. Vac. Sci. Technol. B 23(3), 970 (2005).

${ }^{8}$ F. Liu, K. M. Jones, Y. Xu, W. Nemeth, J. Lohr, J. Neilson, M. J. Romero, M. M. Al-Jassim, and D. L. Young, Adv. Mater. 21, 3002 (2009).

${ }^{9}$ M. Thumm, Plasma Phys. Controlled Fusion 45, A143 (2003).

${ }^{10}$ D. L. Young, P. Stradins, E. Iwaniczko, B. To, B. Reedy, Y. Yan, H. M. Branz, J. Lohr, M. Alvarez, J. Booske, A. Marconnet, and Q. Wang, Mater. Res. Soc. Symp. Proc. 862, A10.5.1 (2005).

${ }^{11}$ R. E. Hummel, Electronic Properties of Materials, 3rd ed. (Springer, 2000), p. 110.

${ }^{12}$ R. F. Pierret, Advanced Semiconductor Fundamentals, 2nd ed. (Prentice Hall, 2002), p. 82-83 \& 182-183.

${ }^{13}$ C. James, Phys. Educ. 23, 358 (1988).

${ }^{14}$ M. N. O. Sadiku, Elements of Electromagnetics, 5th ed. (Oxford University Press, New York, 2009), pp. 436-454.

${ }^{15}$ A. Rehman, High Frequency Electronics 6(4), 18 (2007).

${ }^{16} \mathrm{R}$. Sorrentino and G. Bianchi, Microwave and RF Engineering (Wiley, 2010), p. 31

${ }^{17}$ K. Thompson, Y. B. Gianchandani, J. Booske, and R. F. Cooper, J. Microelectromech. Syst. 11(4), 285 (2002).

${ }^{18}$ S. C. Fong, C. Y. Wang, T. H. Chang, and T. S. Chin, Appl. Phys. Lett. 94, 102104 (2009). 